\title{
ON DERIVATIONS IN SEMISIMPLE BANACH ALGEBRAS
}

\author{
ABDUL NADIM KHAN and MOHD ARIF RAZA \\ Department of Mathematics \\ Faculty of Science \& Arts-Rabigh \\ King Abdulaziz University \\ Kingdom of Saudi Arabia \\ e-mail: abdulnadimkhan@gmail.com \\ arifraza03@gmail.com
}

\begin{abstract}
The objective of the present paper is to prove the following result: Let $A$ be a unital semisimple Banach algebra and $G_{1}$ and $G_{2}$ be open subsets of $A$ such that for each $x \in G_{1}$ and $y \in G_{2}$, there is an integer $n=n(x, y)>1$. If $A$ admits a linear derivation $d: A \rightarrow A$ such that either $d\left((x y)^{n}\right)+d\left(x^{n}\right) d\left(y^{n}\right) \in Z(A)$ or $d\left((x y)^{n}\right)-d\left(x^{n}\right) d\left(y^{n}\right) \in Z(A)$, then $d(A) \subseteq Z(A)$. Moreover, other related results are obtained.
\end{abstract}

\section{Introduction and Main Results}

This research has been motivated by the work of Yood [9]. Throughout this manuscript, $A$ denotes a Banach algebra with identity over $\mathbb{C}, Z(A)$ denotes the center of $A$ and $M$ be a closed linear subspace of $A$. Recall that an algebra $A$ is said to be prime if for any $a, b \in A$,

2010 Mathematics Subject Classification: 16W25, 46J10.

Keywords and phrases: semisimple Banach algebra, linear derivation.

Received December 14, 2017; Revised February 12, 2018

(C) 2018 Scientific Advances Publishers 
$a A b=(0)$ implies $a=0$ or $b=0$ and $A$ is semiprime if for any $a \in A, a A a=(0)$ implies $a=0$. Radical of an algebra is the intersection of all primitive ideals of $A$ and is denoted as $\operatorname{rad}(A)$. An algebra $A$ is called semisimple if $\operatorname{rad}(A)=(0)$. For any $x, y \in A$, the symbol $[x, y]$ will denote the commutator $x y-y x$.

A linear mapping $d: A \rightarrow A$ is said to be a derivation on $A$ if $d(x y)=d(x) y+x d(y)$ holds for all $x, y \in A$. The purpose of this paper is to prove some theorems in this spirit for semisimple Banach algebra involving linear derivation. In [7, 8], Herstein proved that a ring $R$ is commutative if it has no non-zero nilpotent ideal and there is a fixed integer $n>1$ such that $(x y)^{n}=x^{n} y^{n}$ for all $x, y \in R$ (see also [3]). Also it is proved in [2] that a ring is a commutative if $(x y)^{n}=y^{n} x^{n}$ for all $x, y \in R$ and $R$ has the property that $n(n+1)[x, y]=0$ implies $[x, y]=0$ for all $x, y \in R$. In the case of Banach algebra Yood [9] sharpened these results. More precisely, he proved the following result:

Theorem 1.1 ([9], Theorem). Suppose that there are non-empty open subsets $G_{1}$ and $G_{2}$ of $A$ such that for each $x \in G_{1}$ and $y \in G_{2}$, there is an integer $n=n(x, y)>1$, where either $(x y)^{n}-x^{n} y^{n}$ or $(x y)^{n}-y^{n} x^{n}$ lies in $M$. Then $[x, y] \in M$ for all $x, y \in A$.

This result motivated us to prove following theorem:

Theorem 1.2. Let $A$ be a unital semisimple Banach algebra and $G_{1}$ and $G_{2}$ be open subsets of $A$ such that for each $x \in G_{1}$ and $y \in G_{2}$, there is an integer $n=n(x, y)>1$. If $A$ admits a linear derivation $d: A \rightarrow A$ such that either $d\left((x y)^{n}\right)+d\left(x^{n}\right) d\left(y^{n}\right) \in Z(A)$ or $\quad d\left((x y)^{n}\right)-d\left(x^{n}\right)$ $d\left(y^{n}\right) \in Z(A)$, then $d(A) \subseteq Z(A)$. 
Proof. Fix $x \in G_{1}$ and for each $n>1$ we define the set $U_{n}=\{y \in$ $A \mid d\left((x y)^{n}\right)+d\left(x^{n}\right) d\left(y^{n}\right) \notin Z(A)$ and $\left.y \in A \mid d\left((x y)^{n}\right)-d\left(x^{n}\right) d\left(y^{n}\right) \notin Z(A)\right\}$. It is easy to see that $U_{n}$ is open. Thus, by Baire category theorem, if every $U_{n}$ is dense then their intersection is also dense, which contradicts the existence of $G_{2}$. Hence there exists a positive integer $r>1$ such that $U_{r}$ is not dense. Therefore, there exists a non-empty open set $G_{3}$ in the complement of $U_{r}$ such that either $d\left((x y)^{r}\right)+d\left(x^{r}\right) d\left(y^{r}\right) \in Z(A)$ or $d\left((x y)^{r}\right)-d\left(x^{r}\right) d\left(y^{r}\right) \in Z(A)$ for all $y \in G_{3}$. Let $v_{0} \in G_{3}$ and $w \in A$. Then $v_{0}+t w \in G_{3}$ for all sufficiently small real $t$. Then, for each such $t$

$$
d\left(\left(x\left(v_{0}+t w\right)\right)^{r}\right)+d\left(x^{r}\right) d\left(\left(v_{0}+t w\right)^{r}\right) \in Z(A)
$$

or

$$
d\left(\left(x\left(v_{0}+t w\right)\right)^{r}\right)-d\left(x^{r}\right) d\left(\left(v_{0}+t w\right)^{r}\right) \in Z(A),
$$

for $v_{0} \in G_{3}$ and $w \in A$. Thus at least one of (1.1) and (1.2) is valid for infinitely many $t$. Suppose (1.1) holds for these $t$. Then the expression $d\left(\left(x\left(v_{0}+t w\right)\right)^{r}\right)+d\left(x^{r}\right) d\left(\left(v_{0}+t w\right)^{r}\right)$ can be written as

$$
\begin{aligned}
& d\left(A_{r, 0}\left(x, v_{0}, w\right)\right)+d\left(x^{r}\right) B_{r, 0}\left(v_{0}, w\right) \\
& \quad+d\left(A_{r-1,1}\left(x, v_{0}, w\right)\right)+d\left(x^{r}\right) B_{r-1,1}\left(v_{0}, w\right) t \\
& \quad+\ldots \\
& \quad+d\left(A_{1, r-1}\left(x, v_{0}, w\right)\right)+d\left(x^{r}\right) B_{1, r-1}\left(v_{0}, w\right) t^{r-1} \\
& \quad+d\left(A_{0, r}\left(x, v_{0}, w\right)\right)+d\left(x^{r}\right) B_{0, r}\left(v_{0}, w\right) t^{r}
\end{aligned}
$$

where $A_{i, j}\left(x, v_{0}, w\right)$ denotes the sum of all terms in which $x v_{0}$ appears exactly $i$ times and $x w$ appears exactly $j$ times in the expansion of $d\left(x\left(v_{0}+t w\right)^{r}\right)$, where $i$ and $j$ are non-negative integers such that 
$i+j=r$. Similarly, $B_{i, j}\left(v_{0}, w\right)$ is sum of all terms in which $v_{0}$ appears exactly $i$ times and $w$ appears exactly $j$ times in the expansion of $d\left(\left(v_{0}+t w\right)^{r}\right)$, where $i$ and $j$ are nonnegative integers such that $i+j=r$. The above expression is a polynomial in $t$ and the coefficient of $t^{r}$ in this polynomial is $d\left((x w)^{r}\right)+d\left(x^{r}\right) d\left(w^{r}\right)$. Hence $d\left((x w)^{r}\right)+d\left(x^{r}\right) d\left(w^{r}\right) \in Z(A)$. If (1.2) is holds for these $t$ then, we are forced to conclude that $d\left((x w)^{r}\right)-d\left(x^{r}\right) d\left(w^{r}\right) \in Z(A)$. Thus, given $x \in G_{1}$ there is a positive integer $r>1$ depending on $w$ such that for each $w \in A$ either $d\left((x w)^{r}\right)$ $+d\left(x^{r}\right) d\left(w^{r}\right) \in Z(A)$ or $d\left((x w)^{r}\right)-d\left(x^{r}\right) d\left(w^{r}\right) \in Z(A)$. Next, fix $y \in A$ and for each positive integer $k>1$, set $V_{k}=\left\{v \in A \mid d\left((v y)^{k}\right)+d\left(v^{k}\right)\right.$ $d\left(y^{k}\right) \notin Z(A)$ and $\left.d\left((v y)^{k}\right)-d\left(v^{k}\right) d\left(y^{k}\right) \notin Z(A)\right\}$. It is easy to see that each $V_{k}$ is open. If each $V_{k}$ is dense, then by Baire category theorem so is intersection also but this is contrary to what was shown earlier concerning the open set $G_{1}$. Hence there is an integer $m>1$ and a nonempty open subset $G_{4}$ in the complement of $V_{m}$. If $x_{0} \in G_{4}$ and $u \in A$, then $x_{0}+t u \in G_{4}$ for all sufficiently small real $t$. Hence for positive integer $m>1$ either $d\left(\left(\left(x_{0}+t u\right) y\right)^{m}\right)+d\left(\left(x_{0}+t u\right)^{m}\right) d\left(y^{m}\right) \in Z(A)$ or $d\left(\left(\left(x_{0}+t u\right) y\right)^{m}\right)-d\left(\left(x_{0}+t u\right)^{m}\right) d\left(y^{m}\right) \in Z(A)$ for each $u \in A$ and $x_{0} \in G_{4}$. Arguing as above we see that either $d\left((u y)^{m}\right)+d\left(u^{m}\right) d\left(y^{m}\right) \in Z(A)$ or $d\left((u y)^{m}\right)-d\left(u^{m}\right) d\left(y^{m}\right) \in Z(A)$ for each $u \in A$.

Now $S_{k}, k>1$, be the set of $y \in A$ such that for each $w \in A$ either $d\left((w y)^{k}\right)+d\left(w^{k}\right) d\left(y^{k}\right) \in Z(A)$ or $d\left((w y)^{k}\right)-d\left(w^{k}\right) d\left(y^{k}\right) \in Z(A)$, then the union of $S_{k}$ will be $A$. It can be easily proved that each $S_{k}$ is closed. Hence again by Baire category theorem some $S_{l}$ must have a non empty open subset $G_{5}$. Let $y_{0} \in G_{5}$ and $z \in A$, for all sufficiently small real $t$ 
and each $w \in A$ either $d\left(\left(w\left(y_{0}+t z\right)\right)^{l}\right)+d\left(w^{l}\right) d\left(\left(y_{0}+t z\right)^{l}\right) \in Z(A)$ or $d\left(\left(w\left(y_{0}+t z\right)\right)^{l}\right)-d\left(w^{l}\right) d\left(\left(y_{0}+t z\right)^{l}\right) \in Z(A)$. By earlier arguments, we have for each $w, z \in A$ either $d\left((w z)^{l}\right)+d\left(w^{l}\right) d\left(z^{l}\right) \in Z(A)$ or $d\left((w z)^{l}\right)$ $-d\left(w^{l}\right) d\left(z^{l}\right) \in Z(A)$. Next, since $A$ is unital then, for all real $t$ either $d\left(((e+t x) y)^{n}\right)+d\left((e+t x)^{n}\right) d\left(y^{n}\right) \in Z(A)$ or $d\left(((e+t x) y)^{n}\right)-d\left((e+t x)^{n}\right)$ $d\left(y^{n}\right) \in Z(A)$ for all $x, y \in A$. Hence taking coefficient of $t$ in the expansion of above equations, we get either

$$
d\left(x y^{n}+\sum_{k=1}^{n-1} y^{k} x y^{n-k}\right)+n d(x) d\left(y^{n}\right) \in Z(A),
$$

or

$$
d\left(x y^{n}+\sum_{k=1}^{n-1} y^{k} x y^{n-k}\right)-n d(x) d\left(y^{n}\right) \in Z(A)
$$

for all $x, y \in A$. Now, taking $d\left[(y(e+t x))^{n}\right]$ in instead of $d\left[((e+t x) y)^{n}\right]$, we see that either

$$
d\left(y^{n} x+\sum_{k=1}^{n-1} y^{k} x y^{n-k}\right)+n d\left(y^{n}\right) d(x) \in Z(A),
$$

or

$$
d\left(y^{n} x+\sum_{k=1}^{n-1} y^{k} x y^{n-k}\right)-n d\left(y^{n}\right) d(x) \in Z(A),
$$

for all $x, y \in A$. Then at least one of pair of equations $\{(1.3),(1.5)\},\{(1.3)$, $(1.6)\},\{(1.4),(1.5)\}$, and $\{(1.4),(1.6)\}$ must hold. Then, we get

$$
d\left(\left[x, y^{n}\right]\right) \pm n\left[d(x), d\left(y^{n}\right)\right] \in Z(A),
$$


for all $x, y \in A$. Replace $y$ by $e+t y$ in above equation and using same arguments as we have used above, we get

$$
d([x, y]) \pm n[d(x), d(y)] \in Z(A),
$$

for all $x, y \in A$. First we assume that

$$
d([x, y])+n[d(x), d(y)] \in Z(A),
$$

for all $x, y \in A$. Replace $y$ by $y w$ in above expression, we obtain

$$
\begin{aligned}
d([x, y]) w & +[x, y] d(w)+n d(y)[d(x), w] \\
& +n[d(x), d(y)] w+n[d(x), y] d(w) \\
& +n y[d(x), d(w)] \in Z(A),
\end{aligned}
$$

for all $x, y, w \in A$. Taking $y=e+y$ in (1.10), we obtain

$$
n[d(x), d(w)] \in Z(A),
$$

for all $x, w \in A$. In particular, we have

$$
[[d(x), d(w)], d(w)]=0,
$$

for all $x, w \in A$. Then, by Kleinecke-Shirokov theorem ([4], Proposition 13, p. 91) each $z=[d(x), d(w)]$ is a generalized nilpotent element of $A$, i.e., $\rho(z)=\left\|z^{n}\right\|^{1 / n}=0$. Therefore, $[d(x), d(w)]$ is a generalized nilpotent element in $Z(A)$ and so is in radical of $Z(A)$. Since $A$ is semisimple, so $Z(A)$ will also semisimple. Therefore, we conclude that

$$
[d(x), d(w)]=0
$$

for all $x, w \in A$. Next, replace $w$ by $y a$ in (1.13), we get

$$
d(y)[d(x), a]+[d(x), y] d(a)=0
$$


for all $a, x, y \in A$. Taking $a=x$ in above expression, we get

$$
d(y)[d(x), x]+[d(x), y] d(x)=0,
$$

for all $x, y \in A$. Again, replace $y$ by $x y$ in (1.15), we obtain

$$
d(x) y[d(x), x]+[d(x), x] y d(x)=0,
$$

for all $x, y \in A$. In view of ([5], Lemma 4), we have

$$
[d(x), x] y d(x)=0,
$$

for all $x, y \in A$. In particular, it follows that $[d(x), x] y[d(x), x]=0$ for all $x \in A$. Since $A$ is semisimple, the last expression yields that $[d(x), x]=0$. Hence, $d(A) \subseteq Z(A)$.

In a similar manner, we can prove that the same conclusion holds for the case $d([x, y])-[d(x), d(y)] \in Z(A)$ for all $x, y \in A$. Thereby the proof of the theorem is completed.

For unital prime Banach algebra, we can write the following:

Corollary 1.1 ([1], Theorem 1.3). Let $A$ be a unital prime Banach algebra and $G_{1}$ and $G_{2}$ be open subsets of $A$ such that for each $x \in G_{1}$ and $y \in G_{2}$, there is an integer $n=n(x, y)>1$. If A admits a continuous linear derivation $d: A \rightarrow A$ such that either $d\left((x y)^{n}\right)+d\left(x^{n}\right) d\left(y^{n}\right) \in Z(A)$ or $d\left((x y)^{n}\right)-d\left(x^{n}\right) d\left(y^{n}\right) \in Z(A)$, then $A$ is commutative.

Theorem 1.3. Let $A$ be a unital semisimple Banach algebra and $G_{1}$ and $G_{2}$ be open subsets of $A$ such that for each $x \in G_{1}$ and $y \in G_{2}$, there is an integer $n=n(x, y)>1$. If $A$ admits a linear derivation $d: A \rightarrow A$ such that either $d\left((x y)^{n}\right)+x^{n} y^{n} \in Z(A)$ or $d\left((x y)^{n}\right)-x^{n} y^{n} \in Z(A)$, then $d(A) \subseteq Z(A)$. 
Proof. Proceeding on same lines as in case of Theorem 1.1, we arrive at either $d\left((x y)^{n}\right)+x^{n} y^{n} \in Z(A)$ or $d\left((x y)^{n}\right)-x^{n} y^{n} \in Z(A)$ for each $x, y \in A$. Next, since $A$ is unital then, for all real $t$ either $d\left(((e+t x) y)^{n}\right)$ $+(e+t x)^{n} y^{n} \in Z(A) \quad$ or $\quad d\left(((e+t x) y)^{n}\right)-(e+t x)^{n} y^{n} \in Z(A) \quad$ for all $x, y \in A$. Hence taking coefficient of $t$ in the expansion of above equations, we get either

$$
d\left(x y^{n}+\sum_{k=1}^{n-1} y^{k} x y^{n-k}\right)+n x y^{n} \in Z(A),
$$

or

$$
d\left(x y^{n}+\sum_{k=1}^{n-1} y^{k} x y^{n-k}\right)+n x y^{n} \in Z(A),
$$

for all $x, y \in A$. Now, taking $d\left[(y(e+t x))^{n}\right]$ in instead of $d\left[((e+t x) y)^{n}\right]$, we see that either

$$
d\left(y^{n} x+\sum_{k=1}^{n-1} y^{k} x y^{n-k}\right)+n y^{n} x \in Z(A),
$$

or

$$
d\left(y^{n} x+\sum_{k=1}^{n-1} y^{k} x y^{n-k}\right)-n y^{n} x \in Z(A),
$$

for all $x, y \in A$. Then at least one of pair of equations $\{(1.18),(1.20)\}$, $\{(1.18),(1.21)\},\{(1.19),(1.20)\}$, and $\{(1.19),(1.21)\}$ must hold. Then we obtain

$$
d\left(\left[x, y^{n}\right]\right) \pm n\left[x, y^{n}\right] \in Z(A),
$$

for all $x, y \in A$. Replace $y$ by $e+t y$ in above equation and using same arguments as we have used above, we get

$$
d([x, y]) \pm n[x, y] \in Z(A),
$$


for all $x, y \in A$. Suppose

$$
d([x, y])+n[x, y] \in Z(A)
$$

for all $x, y \in A$. That is,

$$
[[d(x), y]+[x, d(y)]+n[x, y], x]=0,
$$

for all $x, y \in A$. Replace $y$ by $[y, z]$ in above expression, we get

$$
[d(x),[y, z]]+[x, d[y, z]]+n[x,[y, z]] \in Z(A),
$$

for all $x, y \in A$. That is,

$$
[d(x),[y, z]]+[x, d[y, z]+n[y, z]] \in Z(A),
$$

for all $x, y \in A$. Application of (1.24) yields that

$$
[d(x),[y, z]] \in Z(A)
$$

for all $x, y \in A$. By ([6], Lemma 1$), d(x) \in Z(A)$ for all $x \in A$, i.e., $d(A) \subseteq Z(A)$. Similar conclusion holds for the case $d([x, y])-[x, y] \in Z(A)$ for all $x, y \in A$. This proves the theorem.

For unital prime Banach algebra, we can write the following:

Corollary 1.2 ([1], Theorem 1.2). Let $A$ be a unital prime Banach algebra and $G_{1}$ and $G_{2}$ be open subsets of $A$ such that for each $x \in G_{1}$ and $y \in G_{2}$, there is an integer $n=n(x, y)>1$. If $A$ admits a continuous linear derivation $d: A \rightarrow A$ such that either $d\left((x y)^{n}\right)+x^{n} y^{n} \in Z(A)$ or $d\left((x y)^{n}\right)+x^{n} y^{n} \in Z(A)$, then $A$ is commutative.

\section{Acknowledgement}

The authors wishes to thank the referee for his/her their valuable comments and suggestions. 


\section{References}

[1] S. Ali and A. N. Khan, On commutativity of Banach algebras with derivations, Bull. Aust. Math. Soc. 91(3) (2015), 419-425.

DOI: https://doi.org/10.1017/S0004972715000118

[2] M. Ashraf and M. A. Quadri, On commutativity of rings with some polynomial constraints, Bull. Aust. Math. Soc. 41(2) (1990), 201-206.

DOI: https://doi.org/10.1017/S0004972700018001

[3] H. E. Bell, On a commutativity theorem of Herstein, Arch. Math. (Basel) 21(1) (1970), 265-267.

DOI: https://doi.org/10.1007/BF01220913

[4] F. F. Bonsall and J. Duncan, Complete Normed Algebras, Springer, New York, 1973.

[5] M. Brešar, Jordan derivations on semiprime rings, Proc. Amer. Math. Soc. 104(4) (1988), 1003-1006.

DOI: https://doi.org/10.1090/S0002-9939-1988-0929422-1

[6] M. Hongan, A note on semiprime rings with derivation, Int. J. Math. Math. Sci. 20(2) (1997), 413-415.

DOI: http://dx.doi.org/10.1155/S0161171297000562

[7] I. N. Herstein, Power maps in rings, Michigan Math. J. 8(1) (1961), 29-32.

DOI: https://doi.org/0.1307/mmj/1028998511

[8] I. N. Herstein, A remark on rings and algebras, Michigan Math. J. 10(3) (1963), 269-272.

DOI: https://doi.org/10.1307/mmj/1028998910

[9] B. Yood, On commutativity of unital banach algebras, Bull. Lond. Math. Soc. 23(3) (1991), 278-280.

DOI: https://doi.org/10.1112/blms/23.3.278 